\title{
Quelle évolution conceptuelle pour le SIRS ?
}

\section{What will be the Evolution of SIRS?}

\author{
F. Beloncle $\cdot$ P. Asfar
}

Reçu le 25 novembre 2015 ; accepté le 30 novembre 2015

(C) SRLF et Lavoisier SAS 2015

Si la question est posée, cela suppose qu'il existe intrinsèquement un problème de concept. Pour tenter d'y répondre, il est important de remonter dans le temps et d'étudier les connaissances qui ont abouti à la genèse du concept de SIRS (Systemic Inflammatory Response Syndrome), qui a fait l'objet d'une conférence de consensus fin 1991 [1]. Le syndrome de réponse inflammatoire systémique associe des symptômes en lien avec la réponse de l'immunité innée à une agression. Cette réponse est appelée sepsis lorsqu'elle est secondaire à une infection. Elle est décrite à la fin des années 1980 dans des modèles expérimentaux animaux endotoxiniques et chez des sujets sains soumis à une endotoxinémie. Ainsi furent décrits la cascade de cytokines proinflammatoires et les signes généraux, ainsi que les perturbations cardiovasculaires et les dysfonctions d'organes associées à cette réponse immunitaire déclenchée par une perfusion d'endotoxine. La conférence de consensus de 1991, dont l'objectif était d'harmoniser les définitions de SIRS, sepsis, sepsis sévère et choc septique, et de stratifier les patients en fonction de leur sévérité a fixé les connaissances des réanimateurs. Conformément au concept expérimental de SIRS, nous avons retenu que les formes les plus sévères d'infections étaient liées à une réaction exagérée, voire incontrôlée, de la réponse immunitaire innée. Nous avions une cible, l'inflammation, et il ne restait plus qu'à trouver le traitement idoine pour diminuer la réponse inflammatoire. Il s'en est suivi de nombreux essais cliniques qui visaient à réduire la réponse inflammatoire, comme par exemple l'administration de fortes doses de corticoïdes [2], l'utilisation d'anticorps anti-endotoxines [3] ou anticytokines [4]. Tous ont échoué. Rapidement, dès le milieu des années 1990, le concept de réponse inflammatoire incontrôlée est

\section{F. Beloncle · P. Asfar $(\bowtie)$}

Département de réanimation médicale et de médecine hyperbare, centre hospitalier universitaire Angers ; laboratoire de biologie neurovasculaire et mitochondriale intégrée, CNRS UMR 6214, INSERM U1083, université Angers, PRES L'UNAM, 4 rue Larrey, F-49933 Angers cedex 9, France

e-mail : piasfar@chu-angers.fr remis en cause et une théorie plus complexe que celle initialement développée est proposée. En même temps que se développe la réponse innée inflammatoire, il existe une réponse anti-inflammatoire (CARS Compensatory Antiinflammatory Response Syndrome), qui module l'inflammation, l'état de l'immunité du patient résultant d'un équilibre dynamique, qui dépend de la proportion des deux réponses, inflammatoire et anti-inflammatoire [5]. L'échec des essais cliniques s'expliquait selon cette nouvelle conception par l'inadéquation chronologique entre le niveau de la réponse immunitaire et l'intervention thérapeutique visant à diminuer l'inflammation. En effet, les études précliniques et les études de phase II avaient mis en place une intervention précoce, visant à diminuer la réponse inflammatoire, alors que dans les essais de phase III, les patients étaient éventuellement inclus à une phase plus tardive de leur réponse immunitaire et, donc, une thérapeutique visant à diminuer la réponse immunitaire avait pu s'avérer contre-productive. Cette évolution conceptuelle a été, en apparence, acceptée par la communauté des réanimateurs. En apparence seulement, car nous sommes aujourd'hui, faute d'un saut technologique, dans l'incapacité d'évaluer en urgence le niveau de la réponse immunitaire d'un patient donné. Dès la révision du concept dans les années 1996-97 [5], il devenait difficile de concilier la définition clinique du SIRS (telle que définie par la conférence de consensus de 1991) avec les processus physiopathologiques sous-tendant la réponse immunitaire au cours du sepsis. La révision de la conférence de consensus en 2001 reconnaissait les limitations de la définition du SIRS et suggérait que sa définition future pourrait éventuellement reposer uniquement sur des critères biochimiques et/ou immunologiques [6]. La liste des signes cliniques et biologiques du sepsis, toujours définis par une infection associée à un SIRS, fut allongée, afin de ne pas méconnaître le diagnostic de sepsis. L'évaluation du niveau de la réponse immunitaire (pro- ou anti-inflammatoire) était réservée pour le futur. Dès lors se posait le problème de l'harmonisation entre les concepts (issus des données expérimentales et cliniques) et les définitions (issues des conférences de consensus). Le nouveau concept proposait une réponse immunitaire 
combinant une réponse pro-inflammatoire (SIRS) modulée par une réponse anti-inflammatoire métachrone ou synchrone (CARS), mais la définition du SIRS issue de la conférence de consensus restait identique. Cette ambiguité aurait peut-être pu être levée en remplaçant l'expression systemic inflammatory response syndrome par systemic immune (innate ?) response syndrome pour souligner la composante anti-inflammatoire et le changement de paradigme. Très récemment, a été publiée par Kaukonen et al. une étude dans le New England journal of Medicine évaluant la sensibilité et la validité des critères de SIRS pour définir le sepsis sévère [7]. Le principal résultat de cette étude était que, si deux critères ou plus de la définition de 1991 du SIRS étaient retenus pour poser le diagnostic de sepsis sévère, un huitième des patients seraient exclus. Ces patients exclus ont la même mortalité que les patients ayant au moins deux critères de SIRS. Ce résultat n'est pas surprenant, dans la mesure où les critères de SIRS n'avaient pas été révisés et accordés (harmonisés ?) avec l'évolution du concept de réponse immunitaire innée.

Les critères de SIRS selon la définition de la conférence de consensus avaient le mérite d'être simples et faciles à obtenir. Cependant, ces critères, outre les arguments déjà développés, sont susceptibles d'être influencés par le timing de leur recueil, l'administration de traitements antipyrétiques, l'administration de traitements bradycardisants comme les bétabloquants, les inhibiteurs calciques ou l'amiodarone, mais aussi les traitements corticoïdes et enfin le tabagisme, susceptibles d'influencer la démargination des polynucléaires. Aussi, il n'est pas surprenant de constater le caractère imparfait des critères de SIRS selon la définition retenue par la conférence de consensus de 1991 pour refléter l'état de la réponse immunitaire à l'agression.

Quelle évolution conceptuelle est envisageable ? Les auteurs de la révision de 2001 [6] ont fait des propositions pertinentes, suggérant de doser des marqueurs biochimiques et/ou immunologiques, qui seraient validés prospectivement par des études épidémiologiques. Il y a 15 ans, cela pouvait paraître utopique, mais de nos jours les avancées technologiques permettent d'envisager de tels dosages. Des approches combinées associant des études génomiques (types de réponses individuelles), protéomiques (réponses cytokiniques par exemple) et métabolomiques (marqueurs biochimiques) permettront de caractériser le niveau de la réponse immunitaire des patients et d'envisager une personnalisation des traitements tels que les immunosuppresseurs ou les immunostimulants. Il reste à déterminer lesquels retenir.

\section{Références}

1. Bone RC, Balk RA, Cerra FB, et al (1992) Definitions for sepsis and organ failure and guidelines for the use of innovative therapies in sepsis. Chest 101:1644-55

2. Bone RC, Fischer CJ, Clemmer TP, et al (1987) A controlled clinical trial of high-dose methylprednisolone in the treatment of severe sepsis and septic shock. N Engl J Med 317:653-8

3. Ziegler EJ, Fischer CJ, Sprung CL, et al (1991) Treatment of gram-negative bacteremia and septic shock with HA-JA human monoclonal anti-body against endotoxin; N Engl J Med 324:42936

4. Fisher CJ, Slotman GJ, Opal SM, et al (1994) Initial evaluation of human recombinant interleukin-1 receptor antagonist in the treatment of sepsis syndrome: a randomized open-label, placebo controlled multicenter trial. Crit Care Med 22:12-21

5. Bone RC, Grodzin CJ Balk RA (1997) Sepsis: a new hypothesis for pathogenesis of the disease process. Chest 112:235-43

6. Levy MM Fink MP, Marshall JC, et al (2003) 2001 SCCM/ ESICM/ACCP/ATS/SIS International sepsis definition conference. Crit Care Med 31:1250-6

7. Kaukonen KM, Bailey M, Pilcher D (2015) Systemic inflammatory response syndrome criteria in defining severe sepsis. N Engl J Med 372:1629-38 